SOUZA, L. dos R. et al. Distribuição de sempre-viva (Paepalanthus sp.) e canela-de-ema (Vellozia squamata) em mosaico vegetacional no Parque Nacional da Serra da Canastra, São Roque de Minas, MG

\title{
DISTRIBUIÇÃO DE SEMPRE-VIVA (Paepalanthus sp.) E CANELA-DE-EMA (Vellozia squamata) EM MOSAICO VEGETACIONAL NO PARQUE NACIONAL DA SERRA DA CANASTRA, SÃO ROQUE DE MINAS, MG. ${ }^{1}$
}

\author{
Líliam dos Reis Souza ${ }^{2}$ \\ Bruno Senna Corrêa ${ }^{3}$ \\ Moacir Alves Andrino ${ }^{4}$ \\ Wedley Gonçalves Veloso 5 \\ Éder dos Reis Simões ${ }^{6}$ \\ Jéssica Elias Reis ${ }^{7}$ \\ Guilherme Henrique Rodrigues Rocha ${ }^{8}$ \\ Paulo MárcioVieira Wild 9 \\ Marília Nádia Coutinho Teixeira ${ }^{10}$ \\ Marisa Brandão Rodrigues ${ }^{11}$
}

\begin{abstract}
RESUMO
A Serra da Canastra compreende um mosaico de fitofisionomias do Cerrado, desde florestais e savânicas até campestres. O objetivo deste trabalho foi avaliar a distribuição de sempre-viva (Paepalanthus sp.) e canela-de-ema (Vellozia squamata) num mosaico vegetacional composto predominantemente por campo limpo úmido e ilhas de campo rupestre, na região da nascente do Parque Nacional da Serra da Canastra. Foi utilizada a metodologia de parcelas, estimador de densidade populacional e foram calculados 2 índices de bioindicação (Grau de confiabilidade e Fator de Importância) para avaliação da integridade biótica do ecossistema. Foram obtidos os respectivos índices de Morisita (ID) e bioindicação (GC e FI) para Vellozia

\section{${ }^{1}$ Como citar este artigo:}

SOUZA, L. dos R. et al. Distribuição de sempre-viva (Paepalanthus sp.) e canela-de-ema (Vellozia squamata) em mosaico vegetacional no Parque Nacional da Serra da Canastra, São Roque de Minas, MG. ForScience: revista científica do IFMG, Formiga, v. 5, n. 2, e00285, out. 2017. Edição especial.

\footnotetext{
${ }^{2}$ Mestranda em Sustentabilidade e Tecnologias Ambientais pelo Instituto Federal de Minas Gerais (IFMG) Campus Bambuí. Currículo Lattes: http://lattes.cnpq.br/9656044208862110. E-mail: liliamreisouza@ gmail.com.

${ }^{3}$ Doutorado em Engenharia Florestal pela UFLA e Docente/Orientador do Programa de Mestrado Profissional em Sustentabilidade e Tecnologia Ambiental pelo IFMG - Campus Bambuí. Currículo Lattes: http://lattes.cnpq.br/4682930954371672. E-mail: bruno.senna@gmail.com.

${ }^{4}$ Mestrando em Sustentabilidade e Tecnologias Ambientais pelo IFMG - Campus Bambuí. Currículo Lattes: http://lattes.cnpq.br/2417083219784053. E-mail: moacir.andrino@ hotmail.com.

${ }_{5}^{5}$ Mestrando em Sustentabilidade e Tecnologias Ambientais pelo IFMG - Campus Bambuí. Currículo Lattes: http://lattes.cnpq.br/4820706022434789. E-mail: wedleyveloso@ hotmail.com.

${ }^{6}$ Graduação em Ciências Biológicas pela Universidade de Uberaba. Currículo Lattes: http://lattes.cnpq.br/3099188380769603. E-mail: bio.eder@yahoo.com.br.

${ }^{7}$ Mestranda em Sustentabilidade e Tecnologias Ambientais pelo IFMG - Campus Bambuí. Currículo Lattes: http://lattes.cnpq.br/8056990452056579. E-mail: jessicaeliasreis23@gmail.com.

${ }^{8}$ Mestrando em Sustentabilidade e Tecnologias Ambientais pelo IFMG - Campus Bambuí. Currículo Lattes: http://lattes.cnpq.br/2117352965972841. E-mail: ghrrocha@gmail.com.

9 Especialização em Gestão e Manejo Ambiental de Sistemas Florestais pela UFLA. Currículo Lattes: http://lattes.cnpq.br/9739037525520766. E-mail: paulowild@gmail.com.

${ }^{10}$ Especialização em Citologia pela Universidade Federal de Ouro Preto (UFOP). Currículo Lattes: http:// lattes.cnpq.br/3011555493546896. E-mail: n.coutt@ hotmail.com.

${ }^{11}$ Graduação em Ciências Biológicas pela Faculdade de Filosofia, Ciências e Letras do Alto São Francisco (FASF). Currículo Lattes: http://lattes.cnpq.br/4411943281342813. E-mail: marisabrandaorodrigues@ yahoo. com.br.
} 
SOUZA, L. dos R. et al. Distribuição de sempre-viva (Paepalanthus sp.) e canela-de-ema (Vellozia squamata) em mosaico vegetacional no Parque Nacional da Serra da Canastra, São Roque de Minas, MG

squamata (ID=1,91; GC=5,25; $\mathrm{FI}=28 \%)$ e para Paepalanthus sp. (ID=2,49; GC=5,25; FI=28\%). Para bioindicação, a confiabilidade foi considerada satisfatória, mas o fator de importância não. A distribuição Paepalanthus sp está intrinsecamente relacionada ao tipo e à umidade dos solos presentes no campo limpo úmido. Vellozia squamata demonstra grande sensibilidade e adaptabilidade à formação rupestre. Os processos de polinização registrados (por Hymenoptera, Diptera e Hemiptera) em Vellozia sp. demonstram a importância deste táxon nesta fitofisionomia.

Palavras-chave: Bioindicação. Estratégia ambiental. Sensibilidade.

\section{INTRODUÇÃO}

Os estudos realizados nos campos rupestres brasileiros têm demonstrado que em sua composição florística há um alto índice de endemismo, pois, nestes locais, há a predominância de condições ecológicas muito particulares (ROMERO; NAKAJIMA, 1999).

Os campos rupestres caracterizam-se pela vegetação campestre predominantemente herbácea e arbustiva associada aos afloramentos (CONCEIÇÃO; PIRANI, 2007). Segundo o IBGE, é classificado como refúgio ecológico arbustivo montano. Trata-se geralmente de um habitat com extrema carência de água, já que a sua capacidade de retenção é mínima; as espécies que conseguem sobreviver não ocorrem casualmente, mas sim por fatores relacionados com a sua adaptação a esta carência. Os solos onde se desenvolvem essas formações apresentam pouco poder de retenção de água, pois podem ser arenosos, ou litólicos de natureza quartzítica, ou, ainda, afloramentos rochosos. Por isso, durante grande parte do ano, a vegetação encontra-se adaptada a estresse hídrico (RODELA, 1998). A comunidade de ilhas de vegetação é constituída por agrupamentos de plantas existentes sobre um afloramento rochoso cuja borda é totalmente delimitada por um substrato pétreo e que, fisionomicamente, forma um manto vegetal denso sobre a rocha (WALDEMAR; IRGANG, 2003).

O objetivo deste trabalho foi avaliar a distribuição e o potencial de bioindicação de Sempre-viva (Paepalanthus sp.) e Canela-de-ema (Vellozia squamata) num mosaico vegetacional composto predominantemente por campo limpo úmido e ilhas de campo rupestre, na região da nascente do Parque Nacional da Serra da Canastra.

\section{DESENVOLVIMENTO}

\subsection{Material e métodos}


SOUZA, L. dos R. et al. Distribuição de sempre-viva (Paepalanthus sp.) e canela-de-ema (Vellozia squamata) em mosaico vegetacional no Parque Nacional da Serra da Canastra, São Roque de Minas, MG

O Parque Nacional Serra da Canastra localiza-se a sudoeste do estado de Minas Gerais, numa área de aproximadamente 200.000 hectares. As principais fitofisionomias encontradas são as matas de galeria e campo úmido $\left(19 \mathrm{~km}^{2}\right)$, cerrado sensu strictu $\left(9 \mathrm{~km}^{2}\right)$, campo sujo $\left(59 \mathrm{~km}^{2}\right)$, campo limpo $\left(287 \mathrm{~km}^{2}\right)$, campo rupestre $\left(100 \mathrm{~km}^{2}\right)$ e áreas de reflorestamento $\left(1 \mathrm{~km}^{2}\right)$ (COUTO JR. et al., 2010). A área de estudo foi uma fitofisionomia de campo limpo úmido associado ao campo rupestre, em solos arenosos e litólicos, que podem ser permanentes ou não, variando de acordo com o nível do lençol freático (Coordenadas: 23K - Lat 7760774 m S/Long 0348702 m E) (Figura 1).

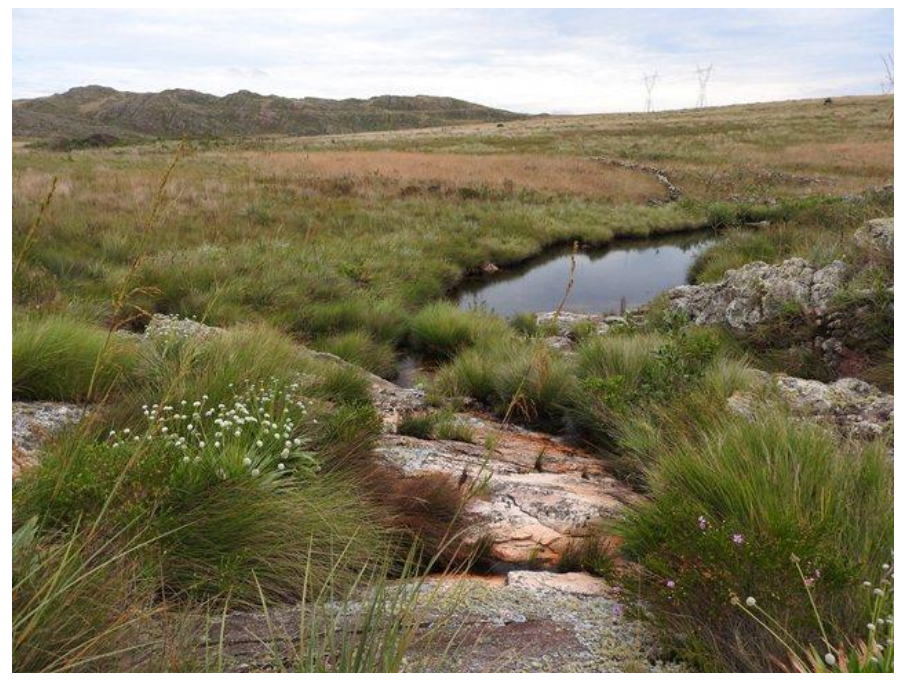

Figura 1 - Área de estudo Fonte: Arquivo pessoal.

Foram analisados indivíduos de Paepalanthus sp. e Vellozia (Figuras 2,3,4,5,6,7).

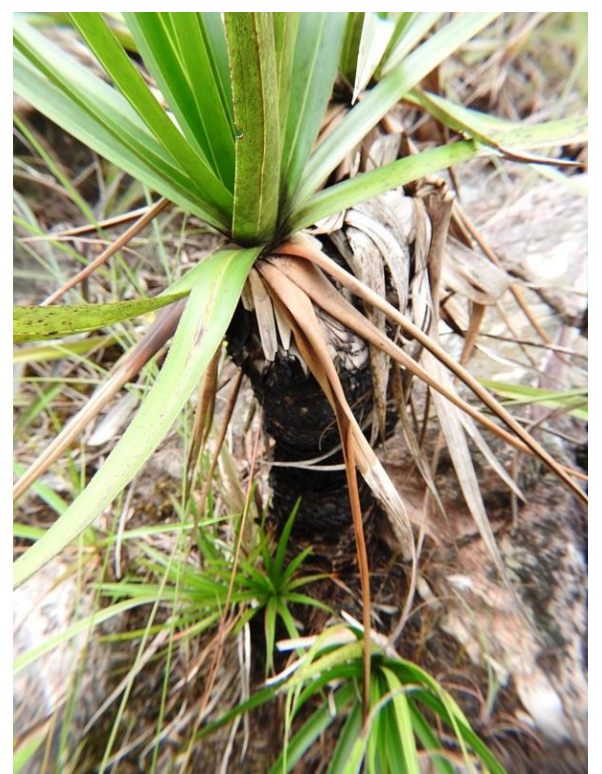

Figura 2 - Sempre-viva (Paepalanthus sp.) Fonte: Arquivo pessoal. 
SOUZA, L. dos R. et al. Distribuição de sempre-viva (Paepalanthus sp.) e canela-de-ema (Vellozia squamata) em mosaico vegetacional no Parque Nacional da Serra da Canastra, São Roque de Minas, MG

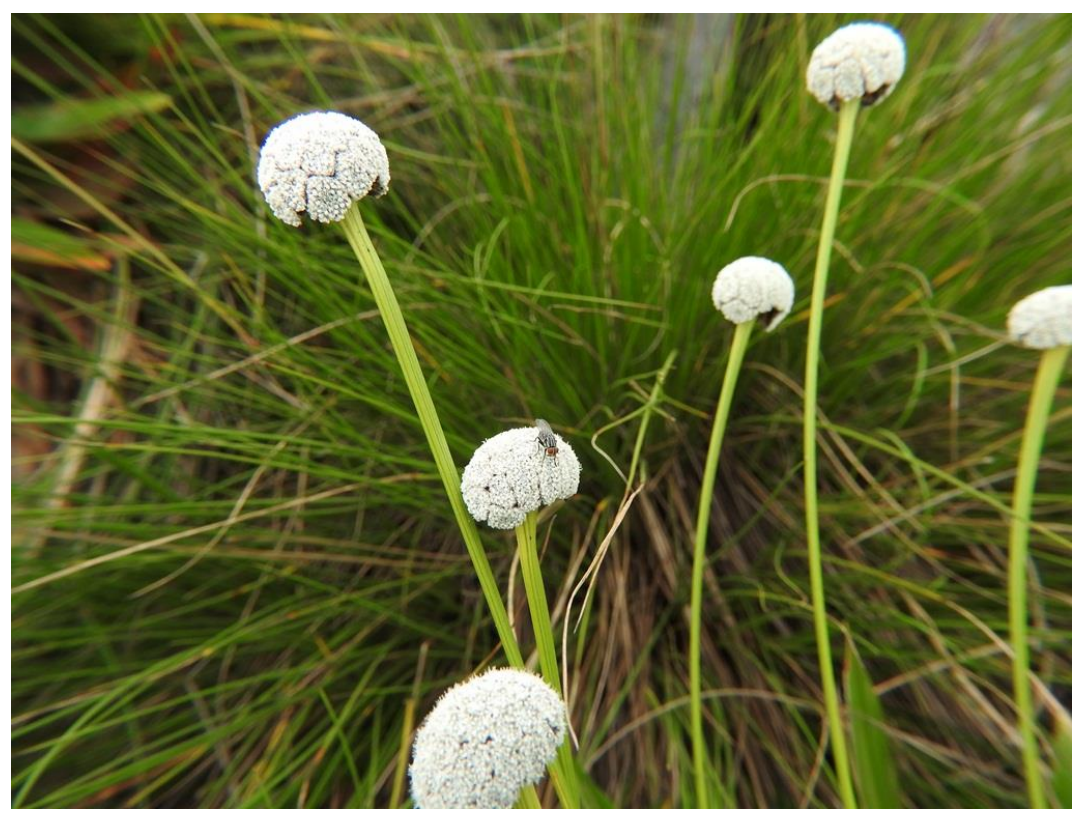

Figura 3 - Sempre-viva (Paepalanthus sp.)

Fonte: Arquivo pessoal.

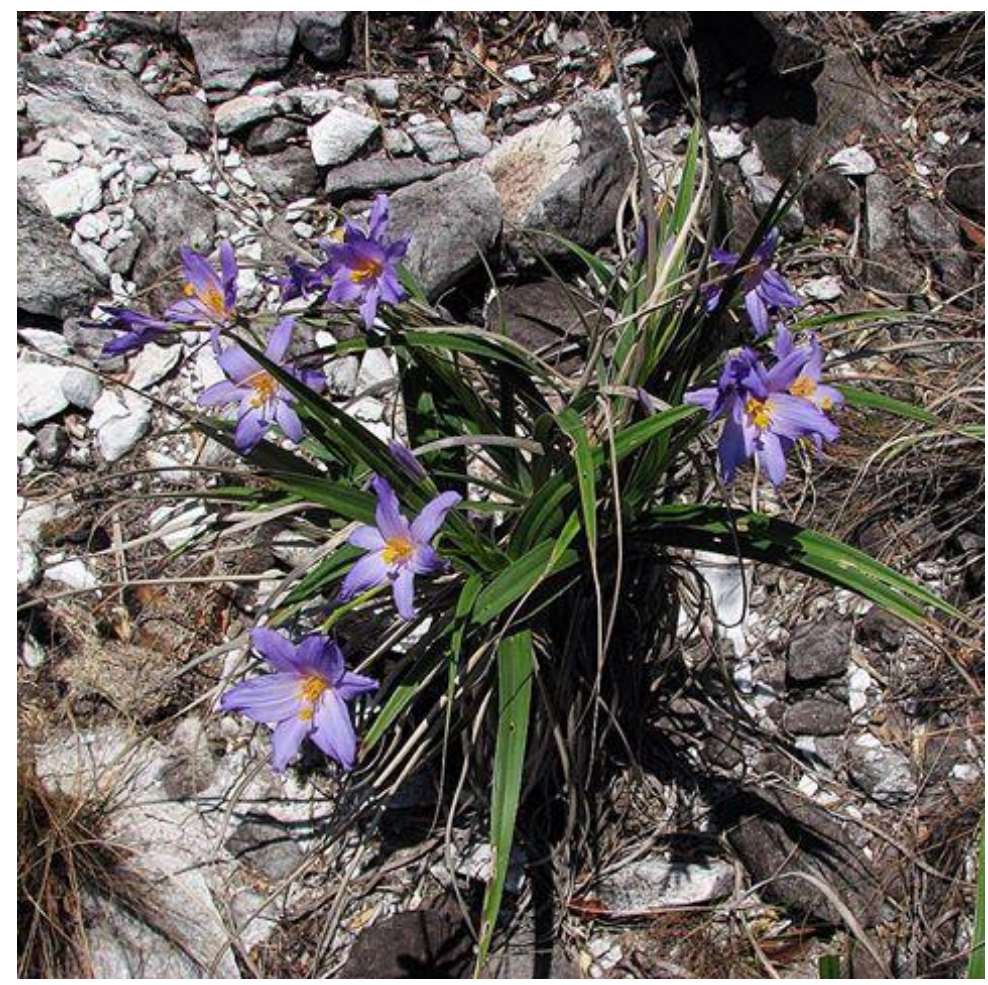

Figura 4 - Canela-de-ema (Vellozia)

Fonte: Arquivo pessoal. 
SOUZA, L. dos R. et al. Distribuição de sempre-viva (Paepalanthus sp.) e canela-de-ema (Vellozia squamata) em mosaico vegetacional no Parque Nacional da Serra da Canastra, São Roque de Minas, MG

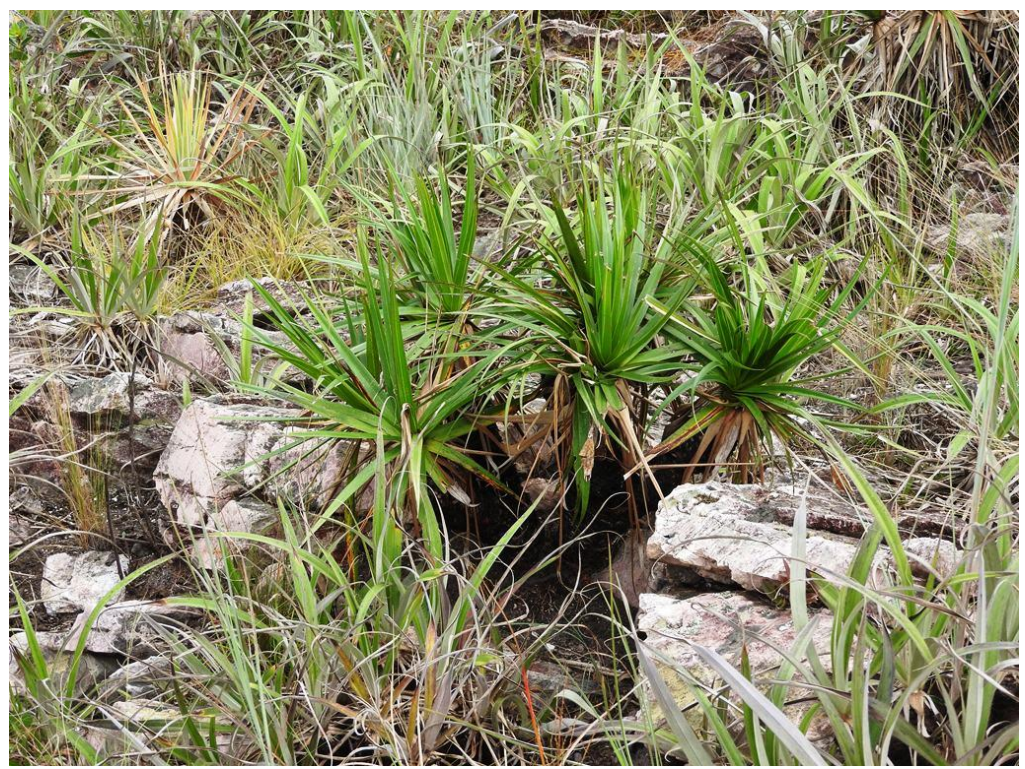

Figura 5 - Canela-de-ema (Vellozia)

Fonte: Arquivo pessoal.

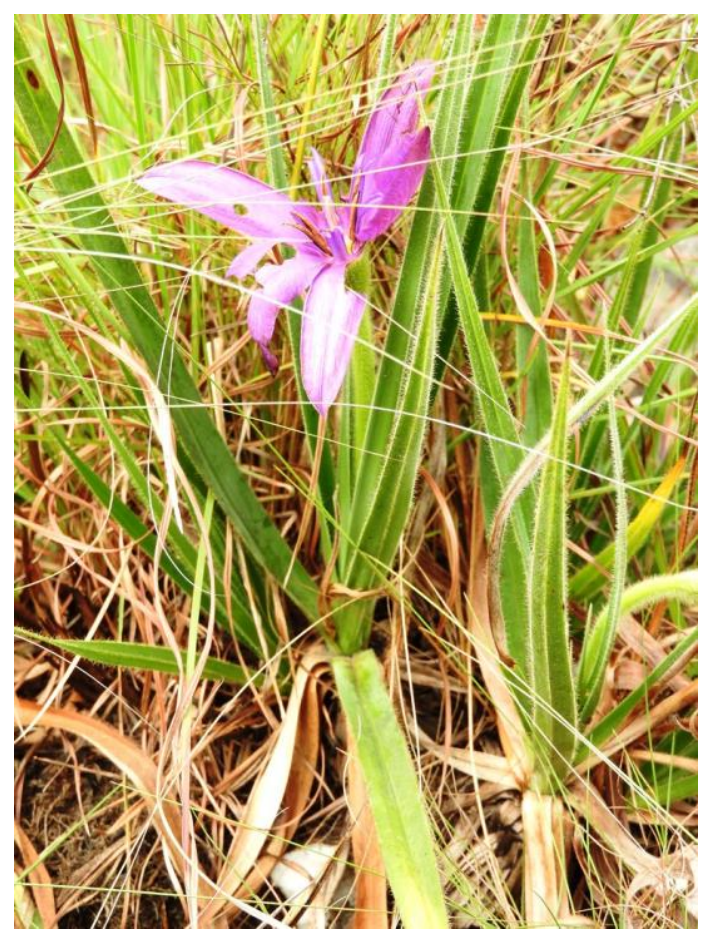

Figura 6 - Canela-de-ema (Vellozia)

Fonte: Arquivo pessoal.

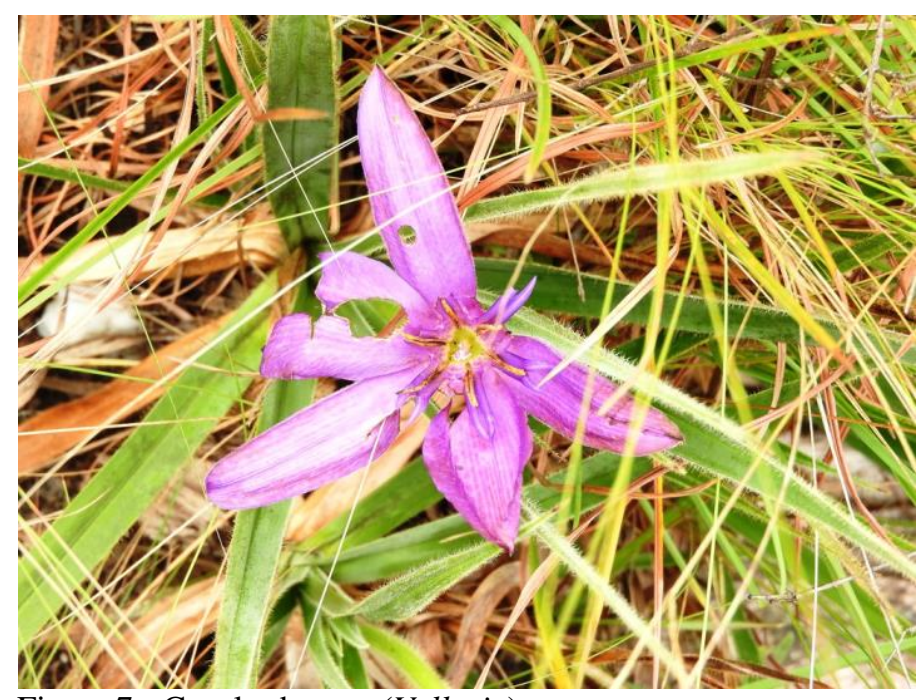

Figura 7 - Canela-de-ema (Vellozia)

Fonte: Arquivo pessoal. 
SOUZA, L. dos R. et al. Distribuição de sempre-viva (Paepalanthus sp.) e canela-de-ema (Vellozia squamata) em mosaico vegetacional no Parque Nacional da Serra da Canastra, São Roque de Minas, MG

O estudo foi realizado em duas parcelas de $50 \times 50 \mathrm{~m}$, alocadas aleatoriamente com subparcelas de $1 \mathrm{~m}^{2}$, totalizando 50 subparcelas (sendo 25 subparcelas em cada parcela), nas quais os indivíduos foram amostrados (Figuras 8 e 9).

P1

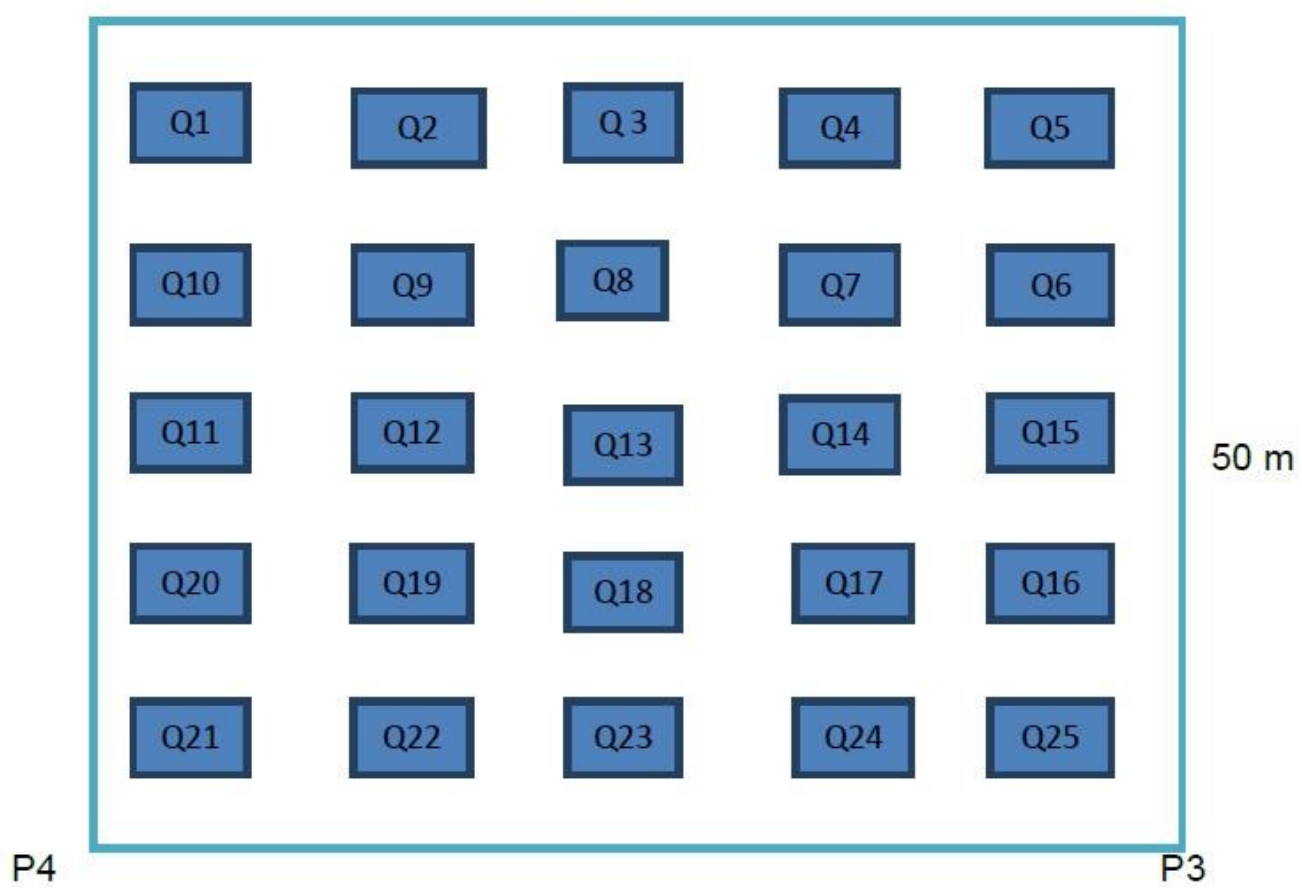

Figura 8 - Apresentação das parcelas amostrais (com subparcelas)

Fonte: Arquivo pessoal.

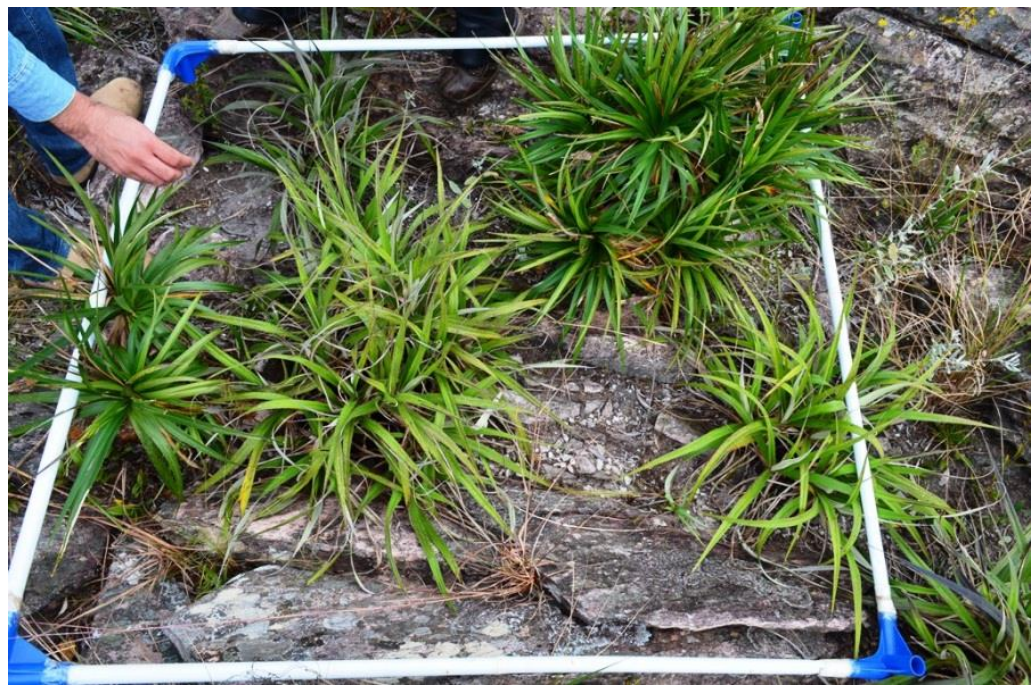

Figura 9 - Amostragem nas subparcelas

Fonte: Arquivo pessoal. 
SOUZA, L. dos R. et al. Distribuição de sempre-viva (Paepalanthus sp.) e canela-de-ema (Vellozia squamata) em mosaico vegetacional no Parque Nacional da Serra da Canastra, São Roque de Minas, MG

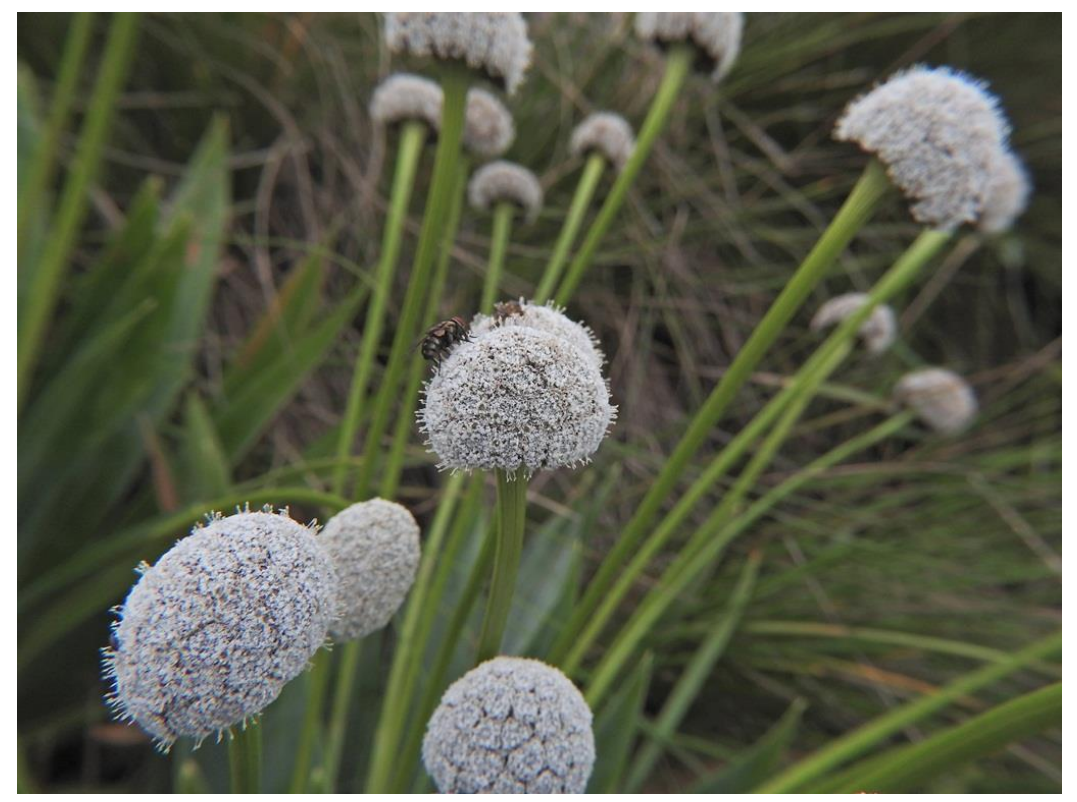

Figura 10 - Interação ecológica entre Paepalanthus sp. e inseto (Diptera) Fonte: Arquivo pessoal.

Os indivíduos foram contabilizados, sendo anotada a sua distribuição espacial e observadas as interações com outros seres vivos (Figuras 10,11,12). A coleta dos dados foi realizada no mês de maio de 2016. As parcelas foram montadas e georreferenciadas.

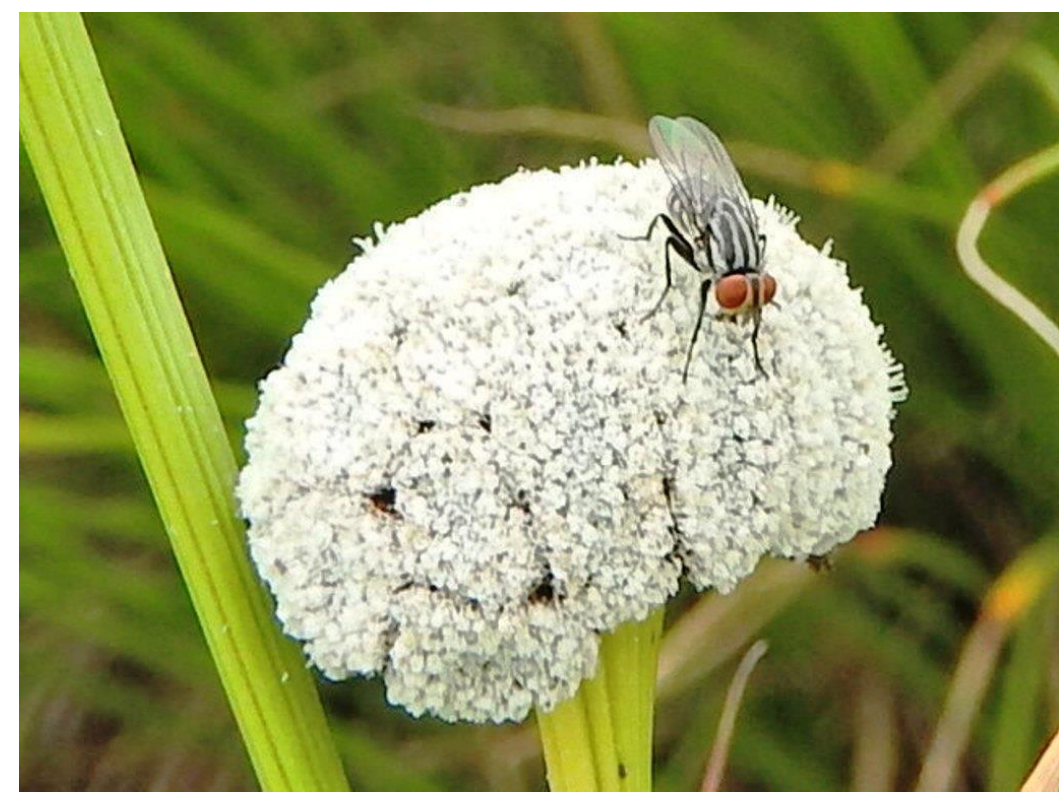

Figura 11- Interação ecológica entre Paepalanthus sp. e inseto (Diptera) Fonte: Arquivo pessoal. 
SOUZA, L. dos R. et al. Distribuição de sempre-viva (Paepalanthus sp.) e canela-de-ema (Vellozia squamata) em mosaico vegetacional no Parque Nacional da Serra da Canastra, São Roque de Minas, MG

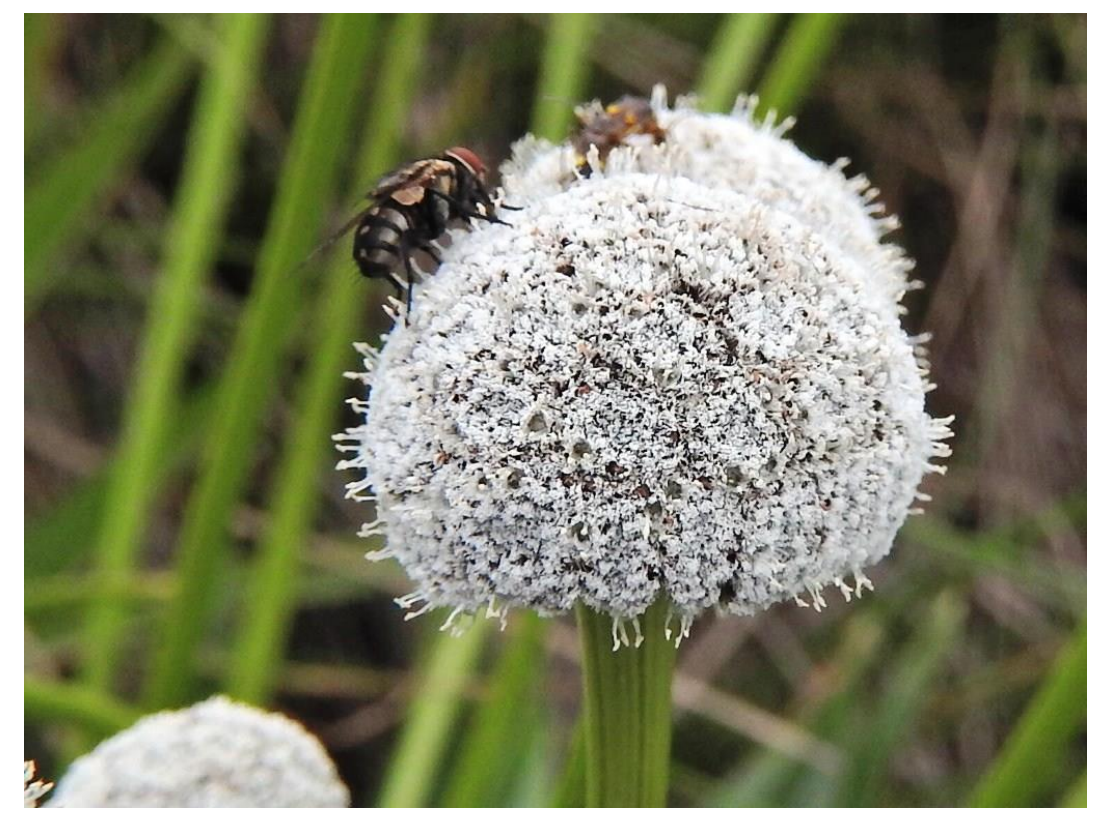

Figura 12 - Interação ecológica entre Paepalanthus sp. e inseto (Diptera) Fonte: Arquivo pessoal.

Foi realizada a análise físico-química de 4 amostras de solo das parcelas e calculadas a Densidade estimada/ha e a Frequência $\left(\mathrm{F}_{\mathrm{A}}\right)$ :

$$
F_{A}=P_{A} / P \times 100
$$

onde: $\mathrm{F}_{\mathrm{A}}=$ frequência da espécie $\mathrm{A} ; \mathrm{P}_{\mathrm{A}}=$ número de amostras ou estações nas quais a espécie A está presente; $\mathrm{P}=$ número total de amostras ou estações, sendo: se $\mathrm{F}=50 \%$ espécie constante; se $10 \%<\mathrm{F}<49 \%$ espécie comum; e, se $\mathrm{F}<10 \%$ espécie rara), índice de Morisita (ID)(dispersão)(distribuição espacial dos indivíduos de uma população):

$$
I D=N\left[\Sigma X^{2}-\Sigma X /(\Sigma X)^{2}-\Sigma X\right]
$$

onde: $\mathrm{N}=$ número total de amostras; $\mathrm{X}=$ somatório do número de indivíduos da mesma espécie em todas as amostras, sendo: A) ID = 1 ao acaso ou aleatória B) ID = 2 agrupada ou contagiosa C) ID = 3 uniforme ou regular). Foram realizadas, ainda, duas análises de bioindicação. A Solidez do indicador foi caracterizada por dois parâmetros (Tabela 1):

1. Confiabilidade: associação entre bioindicador e objeto de bioindicação (fator):

$$
D=N_{1} / N_{2}
$$

2. Fator Importância (FI) mostra quantas vezes o indicador encontra-se com o objeto (\%): 
SOUZA, L. dos R. et al. Distribuição de sempre-viva (Paepalanthus sp.) e canela-de-ema (Vellozia squamata) em mosaico vegetacional no Parque Nacional da Serra da Canastra, São Roque de Minas, MG

$$
F I=N_{2} / N_{1}
$$

sendo: N1 - número de eventos (áreas) quando bioindicador e objeto se encontram, e N2 número de eventos (áreas) quando bioindicador e objeto não se encontram.

Tabela 1- Parâmetros de bioindicação

\begin{tabular}{lcc}
\multicolumn{1}{c}{ Indicador } & $\begin{array}{c}\text { Fator de } \\
\text { Importância } \%\end{array}$ & Confiabilidade \\
\hline Confiável & $>90$ & $>9$ \\
\hline Satisfatório & $75-90$ & $3-9$ \\
\hline Duvidoso & $60-75$ & $1,5-3$ \\
\hline $\begin{array}{l}\text { Indicação } \\
\text { impossível }\end{array}$ & $<60$ & $<1,5$ \\
\hline
\end{tabular}

Fonte: Market et al. (2003).

\subsection{Resultados e Discussão} (Quadro 1).

Os resultados quantitativos das parcelas amostrais podem ser observados abaixo

\begin{tabular}{|c|c|l|}
\hline \multicolumn{3}{|c|}{ Parcela 01: $50 \times 50 \mathrm{~m}$ (Campo limpo úmido) } \\
\hline Quadrante 1×1 m & $\mathrm{N}^{\circ}$ de indivíduos & Espécie \\
\hline Q1 & 03 & Paepalanthus sp. \\
\hline Q2 & 01 & Paepalanthus sp. \\
\hline Q4 & 01 & Paepalanthus sp. \\
\hline Q5 & 01 & Paepalanthus sp. \\
\hline Q3, Q5 - Q25 & 0 & \\
\hline \multicolumn{2}{|c|}{ Parcela 02: 50 x 50 m (Campo rupestre) } \\
\hline Q 1 & 05 & Vellozia squamata \\
\hline Q2 & 03 & Vellozia squamata \\
\hline Q3 & 12 & Vellozia squamata \\
\hline Q4 & 15 & Vellozia squamata \\
\hline Q5 - Q25 & 0 & \\
\hline
\end{tabular}

Quadro 1 - Contagem de indivíduos por parcela

Fonte: Arquivo pessoal.

O resultado da análise de solos (4 amostras) revelou um solo arenoso com ph ácido $(4,6-4,9), \quad \mathrm{P}$ (melh)(fósforo)(1,7-2,9mg/dm $\left.\mathrm{dm}^{3}\right), \quad \mathrm{K} \quad\left(5,0 ; 17,0 ; 34,0 ; 17,0 \mathrm{mg} / \mathrm{dm}^{3}\right), \quad \mathrm{Al}$ $\left(0,78 ; 0,40 ; 0,40 ; 0,94 \quad\right.$ cmolcdm $\left.^{3}\right), \quad M g \quad\left(0.05 ; 0,08 ; 0,02 ; 0,01 \quad \mathrm{mg} / \mathrm{dm}^{3}\right), \quad$ areia $(76,10 \% ; 77,20 \% ; 81,90 \% ; 82,50 \%)$, argila $(3,60 \% ; 4,30 \% ; 2,00 \% ; 5,00 \%)$ e silte 
SOUZA, L. dos R. et al. Distribuição de sempre-viva (Paepalanthus sp.) e canela-de-ema (Vellozia squamata) em mosaico vegetacional no Parque Nacional da Serra da Canastra, São Roque de Minas, MG

$(20,30 \% ; 13,80 \% ; 20,80 \% ; 12,80 \%)$. A densidade estimada (considerando a área total de 200.000 ha do PARNA) para Vellozia squamata foi de 140 ind./ha (sendo 1.400.000 indivíduos (100 km² de campo rupestre)), e, para Paepalanthus sp., foi de 24 ind./ha (sendo 45.600 indivíduos $\left(19 \mathrm{~km}^{2}\right.$ de campo úmido)). O índice de frequência foi de $16 \%$ tanto para Paepalanthus sp. quanto para Vellozia squamata (frequência comum nas áreas de estudo). O padrão de ocorrência pode ser observado na Figura 13.
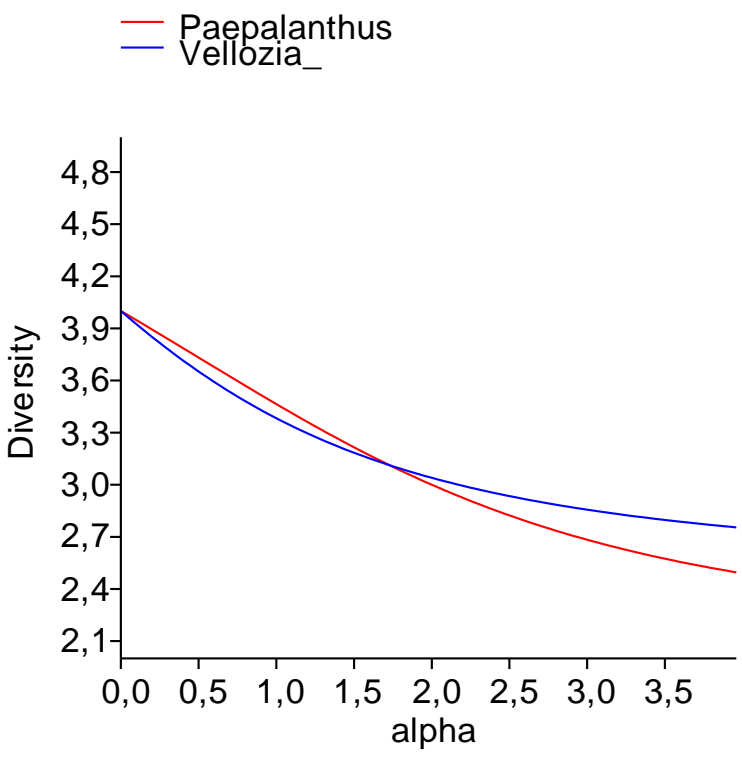

Figura 13 - Padrão de ocorrência dos táxons

Os índices de Morisita foram $\mathrm{ID}=1,91$ para Vellozia squamata e $\mathrm{ID}=2,49$ para Paepalanthus sp. Ambas apresentaram padrão agregado ou sazonal de distribuição (POOLE, 1974). O Índice de Dispersão de Morisita é um dos métodos mais utilizados para a avaliação do padrão de distribuição espacial de indivíduos de espécies vegetais, quando são empregadas parcelas como forma de amostragem (https://dendrolab.wordpress.com/2010/11/10/morisita/). O índice de diversidade beta (Whittaker) foi de 0,25 para ambas as espécies. Os resultados dos índices de bioindicação registraram 5,25 para Confiabilidade (satisfatório) e 28\% para Fator de Importância (indicação impossível) para ambos os táxons (Paepalanthus sp. e Vellozia squamata). Tais resultados estão subestimados pelo número de amostras analisadas. Estudos com bioindicadores servem, em geral, para informar se determinada perturbação tem ou não um impacto biótico, e fornecem informações críticas para a conservação do táxon ou grupo indicador, principalmente quando se sabe que a espécie é rara ou ameaçada (BUTTERFIELD et. al., 1995). Trata-se de uma ferramenta essencial para avaliar a resiliência e a sobrevivência das espécies individuais, visando à conservação de comunidades biológicas inteiras (PRIMACK; RODRIGUES, 2004). McGeoch (1998), bioindicadores são os mais 
SOUZA, L. dos R. et al. Distribuição de sempre-viva (Paepalanthus sp.) e canela-de-ema (Vellozia squamata) em mosaico vegetacional no Parque Nacional da Serra da Canastra, São Roque de Minas, MG

utilizados para demonstrar os efeitos das mudanças ambientais (como alterações no habitat, fragmentação e mudanças climáticas) no sistema biótico, às vezes funcionando, mesmo, como um medidor do estado do ambiente.

\section{CONCLUSÃO}

Os dois táxons estudados possuem estratégias de regeneração após distúrbios (fogo p.ex.). Ambas as espécies são consideradas rebrotadoras ("resprouting”) (VERDU, 2000; BELL, 2001), caracterizando-se por terem gemas em órgãos subterrâneos ou em caules, que permitem a rebrota pós-fogo. Apesar de serem comuns nas fitofisionomias amostradas, o número de amostras não permitiu a apresentação de informações mais robustas. Análises posteriores, associadas à dinâmica do fogo - evento frequente na região - e um banco amostral mais robusto podem fornecer informações mais precisas relacionadas à sensibilidade e resiliência das espécies analisadas.

\section{REFERÊNCIAS}

BELL, D.T. Ecological response syndromes in the flora of Southern Western Australia fire resprouters versus reseeders. The Botanical Review, v. 67, n. 4, p. 417- 440, 2001.

BUTTERFIELD, J. et al. Carabid beatle communities as indicators of conservation potential in upland forest. Forest Ecology and Management v. 79, n.1, p. 63-77, 1995. doi: 10.1016/0378-1127(95)03620-2.

CONCEIÇÃO, A. A; PIRANI, J. R. Diversidade em quatro áreas de campos rupestres na chapada diamantina, Bahia, Brasil: espécies distintas, mas riquezas similares. Rodriguésia. v. 58, n. 1, p. 193-206, 2007.

COUTO JÚNIOR, A. F. et al. Integração de parâmetros morfométricos e imagem Aster para a delimitação das fitofisionomias da Serra da Canastra, Parque Nacional da Serra da Canastra, MG. Revista Brasileira de Geomorfologia, v. 11, n. 1, 2010. Disponível em:

<http://www.lsie.unb.br/rbg/index.php/rbg/article/view/142/136>. Acesso em: 23 jun. 2016.

INSTITUTO CHICO MENDES DE CONSERVAÇÃO DA BIODIVERSIDADE (ICMBIO). Plano de Manejo Parna da Serra da Canastra. Disponível em: <http://www.icmbio.gov.br/ portal/unidadesdeconservacao/biomas-brasileiros/cerrado/unidades-de-conservacao-cerrado/ 2090>. Acesso em: 16 jun. 2016.

MARKET, B. A.; ANTON M. BREURE, A. M.; HARALD; ZECHMEISTER, H.G.

Bioindicators \& Biomonitors: Principles, Concepts, and Applications. vol. 6. Pergamon, 1014 p., June 2003. 
SOUZA, L. dos R. et al. Distribuição de sempre-viva (Paepalanthus sp.) e canela-de-ema (Vellozia squamata) em mosaico vegetacional no Parque Nacional da Serra da Canastra, São Roque de Minas, MG

MCGEOCH, M. A. The selection, testing and application of terrestrial insects as bioindicators. Biological Reviews, v. 73, n. 2, p. 181-201, May 1998.

POOLE, R.W. An introduction to quantitative ecology. New York: McGraw-Hill. 480 p. March 1974.

PRIMACK, R.B.; RODRIGUES, E. Biologia da Conservação. Curitiba: Editora Planta, Brasil, 665 p., 2004.

RODELA, L. G. Cerrados de altitude e campos rupestres do parque Estadual do Ibitipoca, sudeste de Minas Gerais: distribuição e florística por subfisionomias da vegetação. Revista do Departamento de Geografia, n. 12, p 163-189, 1998.

ROMERO, R; NAKAJIMA, J. N. Espécies endêmicas do Parque Nacional da Serra da Canastra, Minas Gerais. Revta brasil. Bot., São Paulo, v. 22, n. 2, p. 259 - 265, Out. 1999.

VERDÚ, M. Ecological and evolutionary differences between Mediterranean seeders and resprouters. Journal of Vegetation Science, v. 11, n. 2, p. 265-268, 2000.

WALDEMAR, C. C.; IRGANG, B. E. A ocorrência do mutualismo facultativo entre Dyckia maritima backer (bromeliaceae) e o cupim Cortaritermes silvestrii (holmgren), nasutitermitinae, em Afloramentos rochosos no parque estadual de Itapuã, Viamão, RS Acta bot. bras. v. 17, n. 1, p. 37- 48, 2003.

\title{
SEMPRE-VIVA (Paepalanthussp.) AND CANELA-DE-EMA (Velloziasquamata) DISTRIBUTION IN VEGETATIONAL MOSAIC IN SERRA DA CANASTRA NATIONAL PARK, SÃO ROQUE DE MINAS, MG, BRAZIL
}

\begin{abstract}
This study aimed to evaluate the distribution of Sempre-viva (Paepalanthus sp.) and Canelade-ema (Vellozia squamata) composed predominantly of moist grassland and of the Serra da Canastra National Park. The plots methodology was used, population density estimator two indexes of bioindication (Degree of Reliability and Importance Factor) were calculated to evaluate the biotic integrity of the ecosystem. The respective Morisita (ID) and bioindicators (GC, FI) indexes for Vellozia squamata $(\mathrm{ID}=1.91 ; \mathrm{CG}=5.25, \mathrm{FI}=28 \%$ ) and Paepalanthus sp. $(\mathrm{ID}=2.49 ; \mathrm{CG}=5.25, \mathrm{FI}=28 \%$ ) were obtained. For bioindication reliability was considered satisfactory, but the importance factor was not. Paepalanthus sp. is intrinsically related to the type and the humidity of the soils present in the moist grassland. Vellozia squamata shows great sensitivity and adaptability to the rock formation. The observed pollination processes (Hymenoptera, Diptera and Hemiptera) in Vellozia demonstrate the importance of this taxon in this phytophysiognomy.
\end{abstract}

Keywords: Bioindication. Environmental strategy. Sensibility.

Submetido em: 24/06/2016

Aprovado em: 15/07/2016

Publicado em: 06/10/2017 